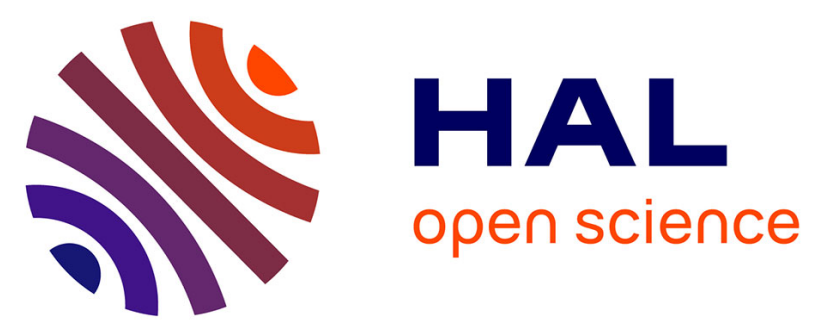

\title{
Attitude control of a gyroscope actuator using event-based discrete-time approach
}

\author{
Bruno Boisseau, Sylvain Durand, John Jairo Martinez Molina, Thibaut \\ Raharijaona, Nicolas Marchand
}

\section{- To cite this version:}

Bruno Boisseau, Sylvain Durand, John Jairo Martinez Molina, Thibaut Raharijaona, Nicolas Marchand. Attitude control of a gyroscope actuator using event-based discrete-time approach. EBCCSP 2015 - 1st IEEE Conference on Event-Based Control Communication and Signal Processing, Jun 2015, Krakow, Poland. 10.1109/EBCCSP.2015.7300677 . hal-01166201

\section{HAL Id: hal-01166201 \\ https://hal.science/hal-01166201}

Submitted on 3 Jul 2015

HAL is a multi-disciplinary open access archive for the deposit and dissemination of scientific research documents, whether they are published or not. The documents may come from teaching and research institutions in France or abroad, or from public or private research centers.
L'archive ouverte pluridisciplinaire HAL, est destinée au dépôt et à la diffusion de documents scientifiques de niveau recherche, publiés ou non, émanant des établissements d'enseignement et de recherche français ou étrangers, des laboratoires publics ou privés. 


\title{
Attitude control of a gyroscope actuator using event-based discrete-time approach
}

\author{
B. Boisseau ${ }^{1}$, S. Durand ${ }^{2}$, J.J. Martinez-Molina ${ }^{1}$, T. Raharijaona ${ }^{2}$, N. Marchand ${ }^{1}$ \\ ${ }^{1}$ Univ. Grenoble Alpes, GIPSA-Lab, F-38000 Grenoble, France \\ CNRS, GIPSA-Lab, F-38000 Grenoble, France \\ 2 Aix-Marseille Université, ISM UMR 7287, 13288, Marseille cedex 09, France
}

E-mail: bruno.boisseau@gipsa-lab.fr

\begin{abstract}
In this paper, a discrete state feedback Linear Quadratic Regulator (LQR) for event-triggered control is presented. To ensure zero steady state error in the case of such controllers, one normally extends the states with an integral action. Instead of using integral action, the idea is to estimate the disturbance causing the steady state error and use this to extend the states. A Lyapunov-based event triggering function is proposed. Practical results using a gyroscope actuator are presented and compared to a classical time-triggered controller. The obtained results demonstrate the simplicity and efficiency of the proposed approach.
\end{abstract}

\section{INTRODUCTION}

With the emergence of the Internet of Things [9], more and more systems are able to be controlled over a network. As these connected objects tend to have weak computational resources it would be interesting to close the control loop remotely. A big challenge is to optimize the battery usage of these objects. Reducing the quantity of the data exchanged seems an interesting approach to improve the battery life by reducing the energy dedicated to communication. One simple way is to increase the sampling time of the closed loop system, but this method faces a bottleneck when the sampling time becomes too close to the system's time constant. Then, instead of using the classical control theory involving periodic triggering paradigm, an event driven control law seems promising. Such an approach consists in computing and updating the control law only when a certain condition is satisfied, another benefit from limiting the number of control updates could be to increase the lifetime of some actuators. Event-triggered control law implies a set of two functions: a feedback function and an event function which determines when the control law has to be updated. Some works have been focused on event-based PID in [2], [5] and [13]. One of the challenges is to find an event condition for MIMO system which guarantees the stability for a given control law. In [3] it is proven that eventtriggered control could ensure the same performances as timetriggered control while requiring less control updates. The majority of research about theoretical stability of the eventtriggered system are based on Lyapunov theory as in [6], [10], [11], [15], and [16]. Some theoretical studies introduce eventtriggered systems with the formalism of hybrid dynamical system, see [14] for an example. A survey of existing methods can be found in [8].

This paper is based on the previous work in [10] designed for nonlinear systems, which leads to an event-based Linear Quadratic Regulator $(L Q R)$ in the linear case. Durand et al. in [4] propose experiments with a gyroscope actuator by considering continuous time system's model. State feedback LQR ensures large stability margins for the closed loop system. In addition, the control input signal is optimal in terms of energy consumption and trajectory which are constrained by weighting factors. Zero steady state error (between the setpoint and the measured output) is ensured using a disturbance observer. In the proposed approach, the event function is designed by considering the linear discrete time model of the system, whereas in [4] the study is done in continuous neglecting the sampling time. The event function ensures that the Lyapunov function of the controlled system will decrease. One of the main interest of considering discrete time system is to avoid the Zeno phenomenon (the occurrence of an infinite number of events in finite time) since a minimal inter sampling interval exists intrinsically. The proposed approach is described as periodic event-triggered control (PETC) in [7], since an event function is computed periodically and indicates if the control input needs to be updated. Moreover, practical results obtained with the gyroscope actuator are shown and discussed in this paper.

The document is organized as follows. In section I the experimental system is presented and its dynamical model is developed. In section II a periodic discrete-time output-feedback is designed, and the disturbance observer is also detailed. In section III the event condition is presented. Experimental results are depicted in IV. Discussions finally conclude the paper.

\section{GYROSCOPIC SYSTEM DESCRIPTION}

Gyroscopes are widely used as actuators, for example to control spacecrafts attitude. The physical principle consists in varying the rotational speed of a flying wheel (motorized gimbal) in order to apply a moment of controlled amplitude (variable-speed single-gimbal gyroscope) or to orientate the axis of the wheel (double-gimbal) to rotate the spacecraft. These devices are generally called control momentum gyroscopes $(\mathrm{CMG})$ and have been a topic of primary interest in control theory.

\section{A. Experimental platform}

The experimental platform in the present work is depicted in Fig. 1. This is a gyroscope M750p from ECP systems [1], where classical LQ control has been previously investigated in [12]. 


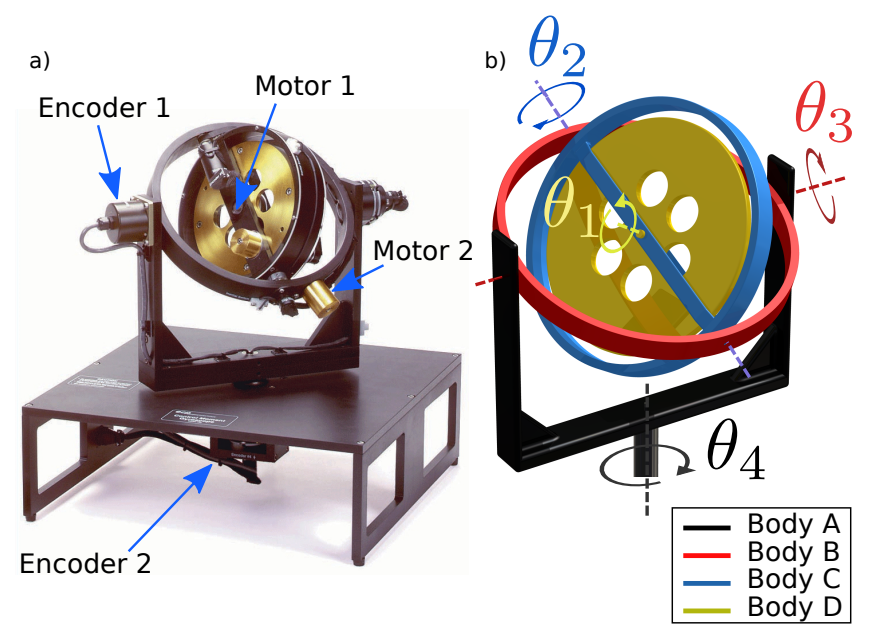

Fig. 1. a) ECP's gyroscope (model M750p), its actuators and sensors b) CAD model of the gyroscope and illustration of the relative angles

1) Electromechanical plant: The gyroscope consists of 4 (rigid) rotating masses. The 4 rigid bodies each has angular position $\theta_{p}$ relative to their rotating gimbal axis $p$, with $p=1,2,3,4$. One can refer to Fig. 1 for a representation of these angles. More precisely, a high inertia brass rotor (body D) is suspended in an assembly with four angular degrees of freedom. The rotor spin torque is provided by a rare earth magnet type DC motor (motor 1) whose angular position is defined as $\theta_{1}$. The first transverse gimbal assembly (body C) is driven by another rare earth motor (motor 2) to effect motion about axis 2 . The relative position between bodies $\mathrm{C}$ and $\mathrm{B}$ is noted as $\theta_{2}$. The subsequent gimbal assembly, body $\mathrm{B}$, rotates with respect to body $\mathrm{A}$ about axis 3 . There is no active torque applied about this axis and the relative angle $\left(\theta_{3}\right)$ is measured by encoder 1 . Similarly, body A rotates without actively applied torque relative to the base frame (inertial ground) along axis 4 . The relative angle $\left(\theta_{4}\right)$ is measured by encoder 2. Two manual brakes may be used to lock the relative position between either bodies A and B or body A and the base frame, in order to reduce the system degrees of freedom.

2) Angular positions and torques: The gyroscope is assumed to be symmetric and the center of all rigid bodies (A, $\mathrm{B}, \mathrm{C}$ and $\mathrm{D}$ ) lie at the center of body $\mathrm{D}$ (the rotor). As a result, only the rotational dynamics need to be taken into account. The following norms are applied hereafter:

- The angular position $\theta_{1}$ of the rotor (body $\mathrm{D}$ ) is not of importance: only the angular velocity $\omega_{1}=\dot{\theta}_{1}$ is considered.

- The angular position $\theta_{2}$ of the rotor drum (body $\mathrm{C}$ ) is set to 0 if the rotor drum (body $\mathrm{C}$ ) is perpendicular to the inner gimbal (body B).

- The angular position $\theta_{3}$ of the inner gimbal (body B) is 0 if the inner gimbal (body B) is perpendicular to the outer gimbal (body A).

- Since the outer gimbal (body A) is able to rotate freely and the gyroscope is assumed to be symmetric, $\theta_{4}$ can be reset to $\theta_{4}=0$ at any angular position of the outer gimbal (body A).
The angular position of the 4 rigid bodies in the gyroscope can be controlled with the 2 internal torques $T_{1}$ and $T_{2}$. These torques are provided by DC motors: $T_{1}$ rotates the $\mathrm{D}$ body around its axis (flying wheel driver) while $T_{2}$ rotates the $\mathrm{C}$ body around the second axis (longitudinal).

\section{B. Dynamic model}

The gyroscope is a complex nonlinear system. However, for a constant angular velocity $\omega_{1}$, it can be modelled as a multivariable linear system. Thus, considering small variations around the operating point defined by the angular speed $\omega_{1}=$ $\Omega$ and the angles $\theta_{p}=0$ for $p=2$ to 4 , gives:

$$
\begin{aligned}
& \dot{\omega}_{2}=\frac{J_{D} \Omega}{I_{C}+I_{D}} \omega_{4}+\frac{1}{I_{C}+I_{D}}\left(T_{2}+d_{2}\right) \\
& \dot{\omega}_{3}=-\frac{1}{J_{B}+J_{C}}\left(T_{1}+d_{1}\right) \\
& \dot{\omega}_{4}=-\frac{J_{D} \Omega}{I_{D}+K_{A}+K_{B}+K_{C}} \omega_{2}
\end{aligned}
$$

where $d_{1}$ and $d_{2}$ are considered as dry friction coefficients. The numerical values of the inertia of the four bodies are $K_{A}=0.067 \mathrm{~kg} \cdot \mathrm{m}^{2}, I_{B}=0.012 \mathrm{~kg} \cdot \mathrm{m}^{2}, J_{B}=0.018 \mathrm{~kg} \cdot \mathrm{m}^{2}$, $K_{B}=0.030 \mathrm{~kg} \cdot \mathrm{m}^{2}, I_{C}=0.0092 \mathrm{~kg} \cdot \mathrm{m}^{2}, J_{C}=0.023 \mathrm{~kg} \cdot \mathrm{m}^{2}$, $K_{C}=0.022 \mathrm{~kg} \cdot \mathrm{m}^{2}, I_{D}=0.015 \mathrm{~kg} \cdot \mathrm{m}^{2}, J_{D}=0.027 \mathrm{~kg} \cdot \mathrm{m}^{2}$. The (fixed) angular velocity for $\omega_{1}$ is $\Omega=42 \mathrm{rad} / \mathrm{s}$. The actuators are limited to:

$$
\begin{aligned}
& \left|T_{1}\right| \leq 0.2 \mathrm{Nm} \\
& \left|T_{2}\right| \leq 3.0 \mathrm{Nm}
\end{aligned}
$$

and the angles are limited to:

$$
\left|\theta_{p}\right| \leq 20^{\circ} \text { for } p=2 \text { to } 4
$$

A state-space representation of the disturbed system (1) in the continuous-time domain is obtained in the form:

$$
\begin{aligned}
& \dot{x}=A_{c} x+B_{c}(u+d) \\
& y=C_{s} x
\end{aligned}
$$

where state, input, disturbance and output vectors are respectively defined by:

$$
\begin{aligned}
& x:=\left(\begin{array}{lllll}
\theta_{3} & \theta_{4} & \omega_{2} & \omega_{3} & \omega_{4}
\end{array}\right)^{T} \\
& u:=\left(\begin{array}{ll}
T_{1} & T_{2}
\end{array}\right)^{T} \\
& d:=\left(\begin{array}{ll}
d_{1} & d_{2}
\end{array}\right)^{T} \\
& y:=\left(\begin{array}{ll}
\theta_{3} & \theta_{4}
\end{array}\right)^{T}
\end{aligned}
$$

and where:

$$
\begin{aligned}
A_{c}= & {\left[\begin{array}{ccccc}
0 & 0 & 0 & 1 & 0 \\
0 & 0 & 0 & 0 & 1 \\
0 & 0 & 0 & 0 & \frac{J_{D} \Omega}{I_{C}+I_{D}} \\
0 & 0 & 0 & 0 & 0 \\
0 & 0 & -\frac{J_{D} \Omega}{I_{D}+K_{A}+K_{B}+K_{C}} & 0 & 0
\end{array}\right], } \\
B_{c}= & {\left[\begin{array}{cc}
0 & 0 \\
0 & 0 \\
0 & \frac{1}{I_{C}+I_{D}} \\
-\frac{1}{J_{B}+J_{C}} & 0 \\
0 & 0
\end{array}\right] C_{s}=\left[\begin{array}{ccccc}
1 & 0 & 0 & 0 & 0 \\
0 & 1 & 0 & 0 & 0
\end{array}\right] }
\end{aligned}
$$

From a control point of view, this system is unstable, it is controllable and observable. 
The discretization of the system defined in (6) with a sampling time $T_{s}$ is computed as follows:

$$
\begin{aligned}
& A=\mathrm{e}^{A_{c} T_{s}}, \\
& B=\left(\int_{0}^{T s} \mathrm{e}^{A_{c} \tau} d \tau\right) B_{c}
\end{aligned}
$$

The resulting discrete-time state space representation is given by:

$$
\begin{aligned}
& x_{k+1}=A x_{k}+B\left(u_{k}+d_{k}\right), \\
& y_{k}=C_{s} x_{k} \quad \text { with } \quad k \in \mathbb{N}
\end{aligned}
$$

where the subscript $\mathrm{k}$ in $x_{k}$ denotes the $k^{\text {th }}$ sample of the vector $x\left(x_{k}=x(k)\right)$.

\section{Problem statement}

The control scheme proposed in this paper is shown Fig. 2.

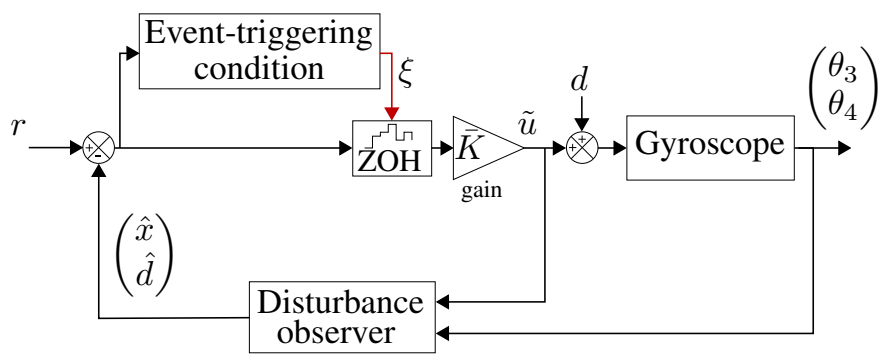

Fig. 2. Event-triggered control strategy of the gyroscope. Since only the angles $\theta_{3}$ and $\theta_{4}$ are measured, a linear observer estimates the states $\hat{x}$ and the dry friction $\hat{d}$ in order to perform the event-based state feedback control.

How can we robustly control the gyroscope's angles $\theta_{3}$ and $\theta_{4}$ to track references $(r)$ taking into account dry friction $(d)$ and reducing the control updates ?

Moreover, one has to find an event-triggering condition $\xi$ which indicates if one needs to update the control signal $\tilde{u}=\bar{K} \cdot\left(\begin{array}{ll}r-\left(\begin{array}{ll}\hat{x} & \hat{d}\end{array}\right)^{T}\end{array}\right)$.

\section{PERIODIC DISCRETE-TIME CONTROL}

\section{A. Observer design}

From Fig. 2 since only $\theta_{3}$ and $\theta_{4}$ are measured, a linear disturbance observer is required for the event-based statefeedback control law. The states considered in (5) are extended as follows:

$$
\bar{x}_{k}=\left(\begin{array}{l}
x_{k} \\
d_{k}
\end{array}\right)
$$

Dry frictions are considered as constant $\left(d_{k+1}=d_{k}\right)$. The augmented discrete-time system becomes:

$$
\begin{aligned}
& \bar{x}_{k+1}=\underbrace{\left[\begin{array}{cc}
A & B \\
0_{2 \times 5} & I_{2}
\end{array}\right]}_{\bar{A}} \bar{x}_{k}+\underbrace{\left[\begin{array}{c}
B \\
0_{2}
\end{array}\right]}_{\bar{B}} u_{k}, \\
& y_{k}=\underbrace{\left[\begin{array}{cc}
C_{s} & 0_{2}
\end{array}\right]}_{\bar{C}} \bar{x}_{k}
\end{aligned}
$$

where $0_{n \times m}\left((n, m) \in \mathbb{N}^{2}\right)$ represents a zero matrix of dimension $n \times m$ and $I_{2}$ represents the identity matrix of dimension two. A linear Luenberger state observer for this system is designed:

$$
\hat{\bar{x}}_{k+1}=\bar{A} \hat{\bar{x}}_{k}+\bar{B} u_{k}+L\left[y_{k}-\bar{C} \hat{\bar{x}}_{k}\right]
$$

where $\hat{\bar{x}}=\left(\begin{array}{ll}\hat{x} & \hat{d}\end{array}\right)^{T}$ is the estimated augmented state vector. The matrix $L$ is calculated to stabilize the error of observation defined by:

$$
\tilde{\bar{x}}_{k}:=\bar{x}_{k}-\hat{\bar{x}}_{k}
$$

An optimal observer can be designed as the dual problem of the LQR state-feedback problem (also known as Kalman filter). The matrix $L$ is finally obtained as:

$$
L:=\left(F+\bar{C}^{T} U \bar{C}\right)^{-1} \bar{C}^{T} U \bar{A}^{T}
$$

where $U$ is positive definite matrix solution of the discrete algebraic Ricatti equation (DARE) given by:

$$
U:=W+\bar{A}\left(U-P \bar{C}^{T}\left(F+\bar{C} U \bar{C}^{T}\right)^{-1} \bar{C} U\right) \bar{A}^{T}
$$

The role of $W$ and $F$, positive definite matrices, consists in establishing a trade-off between the quality of the sensors (sensor noise, measurement bias) and the performances of the actuators (perturbations in the input, friction).

\section{B. Control design}

In the augmented model of the gyroscope (11) two states are neither controllable nor stable. Therefore, it is necessary to consider the following model to design the full state-feedback controller:

$$
\bar{x}_{k+1}=\underbrace{\left[\begin{array}{cc}
A & B \\
0_{2 \times 5} & I_{2} \cdot(1-\epsilon)
\end{array}\right]}_{\bar{A}_{s}} \bar{x}_{k}+\bar{B} u_{k} \text { where } \epsilon \ll 1 \in \mathbb{R}^{+}
$$

The control signal stabilizing the system is as follows:

$$
\begin{aligned}
& u_{k}=-\bar{K} \hat{\bar{x}}_{k} \\
& \text { with } \quad \bar{K}=\left(R+\bar{B}^{T} P \bar{B}\right)^{-1} \bar{B}^{T} P \bar{A}_{s}
\end{aligned}
$$

Where $P$ is a solution of the DARE:

$$
P=Q+\bar{A}_{s}^{T}\left(P-P \bar{B}\left(R+\bar{B}^{T} P \bar{B}\right)^{-1} \bar{B}^{T} P\right) \bar{A}_{s}
$$

This is a classical discrete Linear Quadratic Regulator (LQR) which makes the closed-loop system stable while minimizing the following quadratic cost:

$$
J=\sum_{k=0}^{\infty}\left(\hat{\bar{x}}_{k}^{T} Q \hat{\bar{x}}_{k}+u_{k}^{T} R u_{k}\right)
$$

The first term in (20) corresponds to the energy of the estimated states $\hat{\bar{x}}_{k}$, while the second term corresponds to the energy of the control $u_{k}$. Q and $\mathrm{R}$ are tunable positive definite matrices to weight the importance of the minimization of the states energy cost or the control energy cost. 


\section{Reference tracking}

Only stabilization around zero has been considered in the previous section. Therefore, in order to track a given setpoint, lets define the state error:

$$
e_{k}:=\hat{\bar{x}}_{k}-r_{k}
$$

where $r_{k}$ is the state reference vector and $e_{k}$ is the state error vector. If only slow variation of the reference is considered $\left(r_{k+1}=r_{k}\right)$ we can write:

$$
e_{k+1}=\bar{A} e_{k}+\bar{B} \tilde{u}_{k}
$$

Then the previous controller could stabilize $e_{k}$ around zero which is the same as tracking the setpoint $r_{k}$. The control signal would be:

$$
\tilde{u}_{k}=-\bar{K} e_{k}=\bar{K}\left(r_{k}-\hat{\bar{x}}_{k}\right)
$$

\section{EVENT-BASED DISCRETE-TIME CONTROL}

Previously, a controller has been designed to ensure the closed-loop stability by periodically updating the control signal. Now the problem is to reduce the number of the control signal updates while keeping the closed-loop system stable. So the appropriate instants when the control needs to be refreshed for keeping stability have to be determined.

Lets define an event function $\xi$, that indicates if one needs (when $\xi \geq 0$ ) or not (when $\xi<0$ ) to update the control. The solution of the discrete system (22) with an event-based statefeedback starting in $e_{0}$ at $k=0$ is then defined as the solution of the discrete system:

$$
e_{k+1}=\bar{A} e_{k}-\bar{B} \bar{K} e_{i} \quad \forall k \in[i, i+1[
$$

where the time instants $i$, with $i \in \mathbb{N}$ (determined when the event function $\xi \geq 0$ ) are considered as events and $e_{i}$ is the memory of the state value at the last event. With this strategy, the control signal becomes:

$$
\tilde{u}_{k}= \begin{cases}-\bar{K} e_{k} & \text { if } \xi \geq 0 \\ \tilde{u}_{i} & \text { otherwise }\end{cases}
$$

The aim is to find an event-function $\xi$ which makes the eventtriggered closed-loop system stable. How is construct this event-function is detailed in the sequel. Consider a function $V: \mathbb{R}^{7} \rightarrow \mathbb{R}$ defined by:

$$
V_{k}:=e_{k}^{T} P e_{k}
$$

where the matrix $P$ satisfies the DARE (19). This function is a Control Lyapunov Function (CLF) for system (22), and consequently for system (8), since $\tilde{u}_{k}$ in (23) renders $\Delta V=$ $V_{k+1}-V_{k}$ strictly negative for all $k \in \mathbb{N}$.

The idea is to compare the decrement of the Lyapunov function (26) for both cases: when the control is updated with when the control is held constant. The one-step ahead Lyapunov function $V_{k+1}$ is predicted using the one-step tracking error (22) (as $x_{k+1}$ is not known at instant $\mathrm{k}$ ). If the control signal is updated, the decrement of the Lyapunov function will be:

$$
\Delta V=\left((\bar{A}-\bar{B} \bar{K}) e_{k}\right)^{T} P\left((\bar{A}-\bar{B} \bar{K}) e_{k}\right)-e_{k}^{T} P e_{k}
$$

On the other hand, if the control is held constant we have:

$$
\Delta V_{i}=\left(\bar{A} e_{k}-\bar{B} \bar{K} e_{i}\right)^{T} P\left(\bar{A} e_{k}-\bar{B} \bar{K} e_{i}\right)-e_{k}^{T} P e_{k}
$$

The event function is then computed as follows:

$$
\left.\left.\xi\left(e_{k}, e_{i}\right)=\Delta V_{i}-\sigma \Delta V \quad \text { with } \quad \sigma \in\right] 0 ; 1\right]
$$

This is based on [10], where $\sigma$ is a tunable parameter used to weight the difference between $\Delta V$ and $\Delta V_{i}$. The convergence will be faster with higher $\sigma$ value, but with more events in return. Following ideas of [10], the stability is ensured because the Lyapunov function of the event-triggered system is forced to be strictly negative, thanks to the construction of $\xi$ in (29).

\section{EXPERIMENTAL TESTS}

\section{A. Scenario}

The scenario is such that the angular positions of the gyroscope $\theta_{3}$ and $\theta_{4}$ have to track a sinusoidal reference signal. Prior to this, $\omega_{1}$ is stabilized around a constant value to satisfy the assumption of the linear form (1). Different strategies will be compared in this section:

i) Time-triggered observer-based discrete-time LQR:

- with disturbance estimation and rejection.

- without disturbance estimation and rejection.

ii) Event-triggered observer-based discrete-time LQR:

- with disturbance estimation and rejection.

- without disturbance estimation and rejection.

The computation and the real-time control is done with a computer running xPC target ${ }^{\circledR}$ and Matlab Simulink ${ }^{\circledR}$. The chosen sampling time is $T_{s}=20 \mathrm{~ms}$.

\section{B. LQ control synthesis}

The weighting matrices chosen for the controller and the observer are the following (for $\epsilon=10^{-6}$ ):

$$
\begin{aligned}
& Q=\operatorname{diag}\left(\left[\begin{array}{lllllll}
10 & 70 & 1 & 0 & 1 & 0 & 0
\end{array}\right]\right), \\
& R=\operatorname{diag}\left(\left[\begin{array}{ll}
3 & 0.02
\end{array}\right]\right) \text {, } \\
& W=\operatorname{diag}\left(\left[\begin{array}{lllllll}
1 & 1 & 1 & 1 & 1 & 10^{3} & 10^{3}
\end{array}\right]\right) \text {, } \\
& F=\operatorname{diag}\left(\left[\begin{array}{ll}
1 & 1
\end{array}\right]\right)
\end{aligned}
$$

The weighting factors in $\mathrm{R}$ are chosen to respect the actuator saturation while the weighting factors in $\mathrm{Q}$ are chosen to ensure fast convergence of the first two states. The disturbances are quickly estimated thanks to the weighting factors in $\mathrm{W}$.

\section{Performance indexes}

Several performance indicators introduced in [13] are used:

- The number $(\mathrm{Nb})$ of samples required to perform the test bench.

- The integral absolute error (IAE) index, which gives information about the reference tracking performances

$$
I A E=\sum_{k=0}^{n}|e(k)| \cdot T_{s}
$$

- By analogy, the integral absolute control (IAU) index gives information about the control effort

$$
I A U=\sum_{k=0}^{n}|\tilde{u}(k)| \cdot T_{s}
$$

The performance indexes obtained for the experimental results are reported in Table I and discussed in the sequel. 
TABLE I. PERFORMANCE INDEXES OBTAINED WITH DIFFERENT CONTROL STRATEGIES

\begin{tabular}{|l|c|c|c|c|}
\hline \multicolumn{2}{|l|}{} & Nb & IAE & IAU \\
\hline Time-based LQR strategy without dry friction cancellation & 1000 & 1.29 & 2.01 \\
\hline Event-based LQR strategy without dry friction cancellation & $\sigma=0.9$ & 176 & 1.48 & 2.03 \\
& $\sigma=0.4$ & 129 & 1.91 & 2.36 \\
\hline Time-based LQR strategy with dry friction cancellation & 1000 & 0.46 & 2.08 \\
\hline Event-based LQR strategy with dry friction cancellation & $\sigma=0.9$ & $\mathbf{4 2 1}$ & $\mathbf{0 . 4 6}$ & $\mathbf{2 . 0 4}$ \\
& $\sigma=0.4$ & $\mathbf{2 4 6}$ & $\mathbf{0 . 5 3}$ & $\mathbf{2 . 1 7}$ \\
\hline
\end{tabular}

\section{Results}

Experimental results for the time-triggered LQR control with disturbance rejection are detailed in Fig. 3.
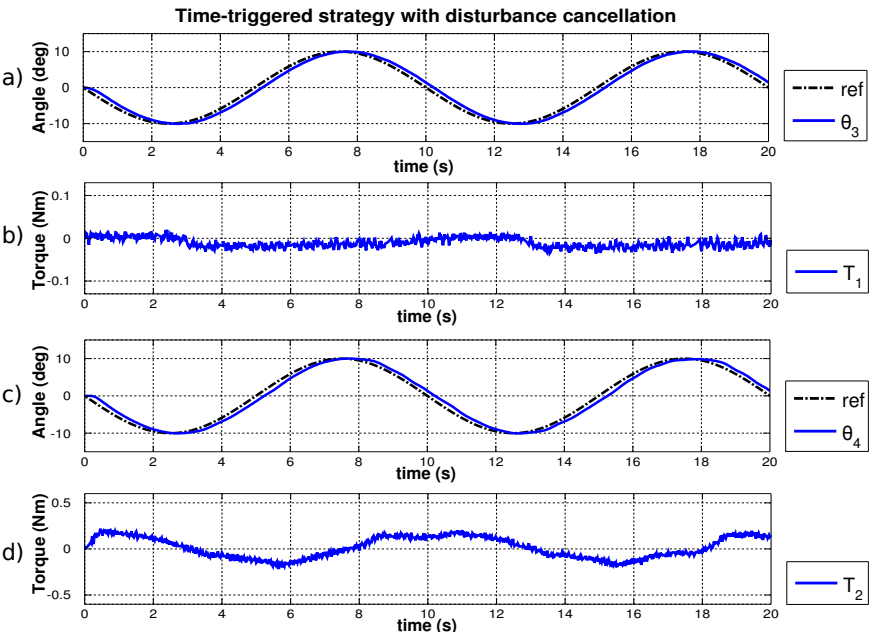

Fig. 3. Experimental results: time-triggered strategy with disturbance rejection

Plot a) illustrates the setpoint and the measured angle $\theta_{3}$, while plot b) shows the applied control $T_{1}$. Plots c) and d) show $\theta_{4}$, the setpoint and the control $T_{2}$. The observer error for $\theta_{3}$ and $\theta_{4}$ are plotted in Fig. 4, these plots illustrate the accuracy of the linear observer.
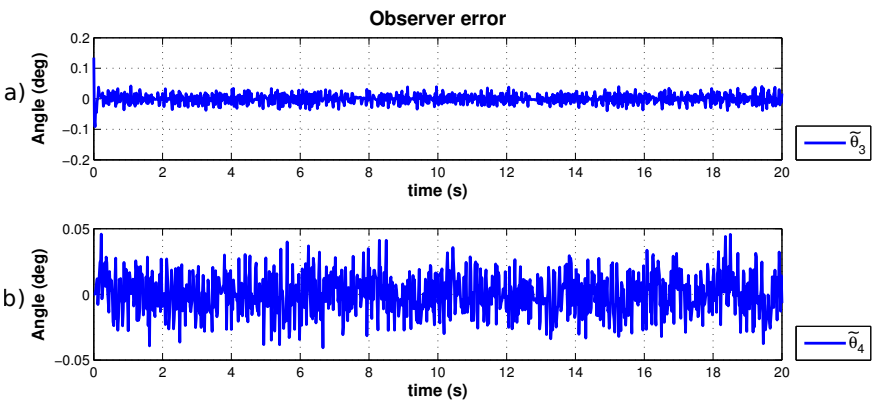

Fig. 4. Experimental results: Observer error

For event-triggered strategies, the results with two different values of $\sigma$ are reported. Fig. 5 and Fig. 6 show event-triggered LQR control with the disturbance estimation and rejection for $\sigma=0.9$ and $\sigma=0.4$ respectively. The extra plot e) indicates when the control input signal is updated, '0' means the control is held and ' 1 ' means the control is updated.
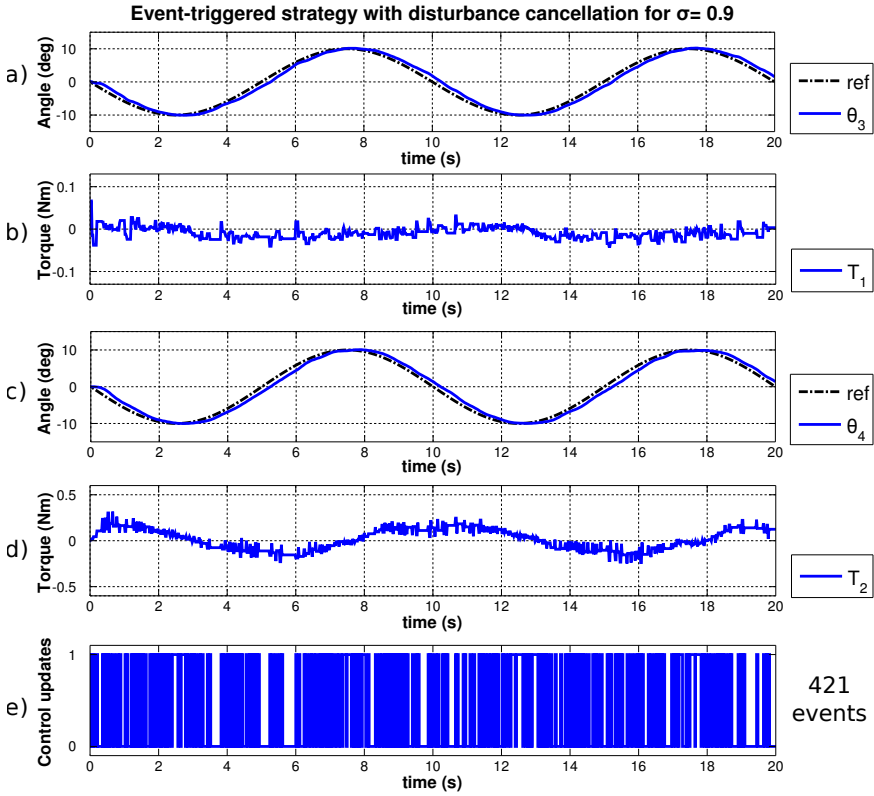

Fig. 5. Experimental results: event-triggered LQR strategy with disturbance rejection for $\sigma=0.9$
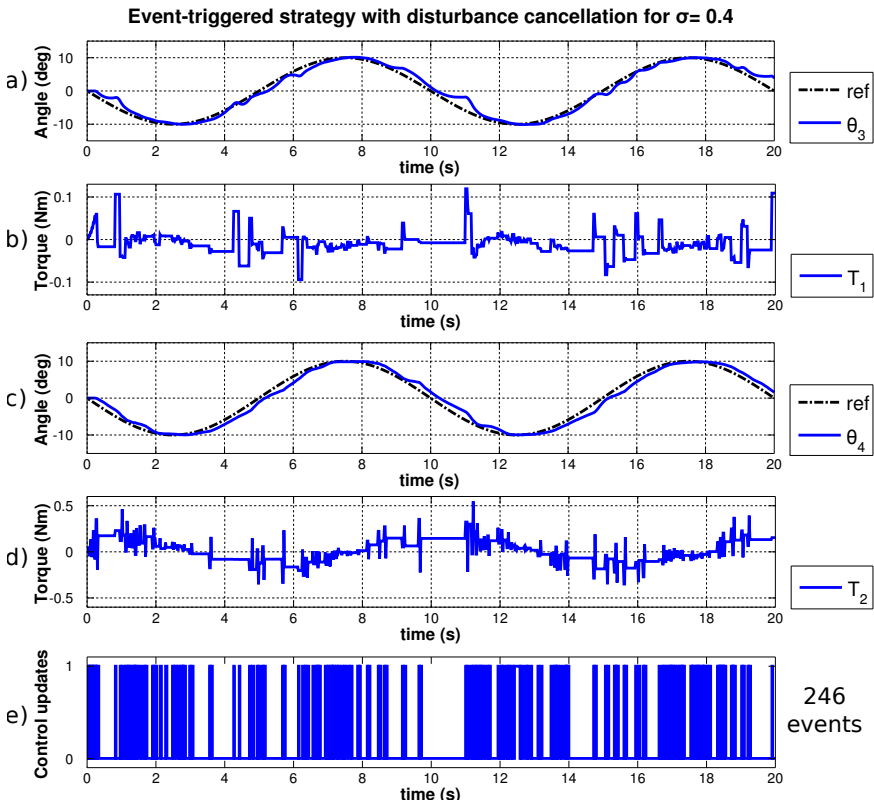

Fig. 6. Experimental results: event-triggered LQR strategy with disturbance rejection for $\sigma=0.4$

The estimation of the dry friction is illustrated in Fig. 7. 

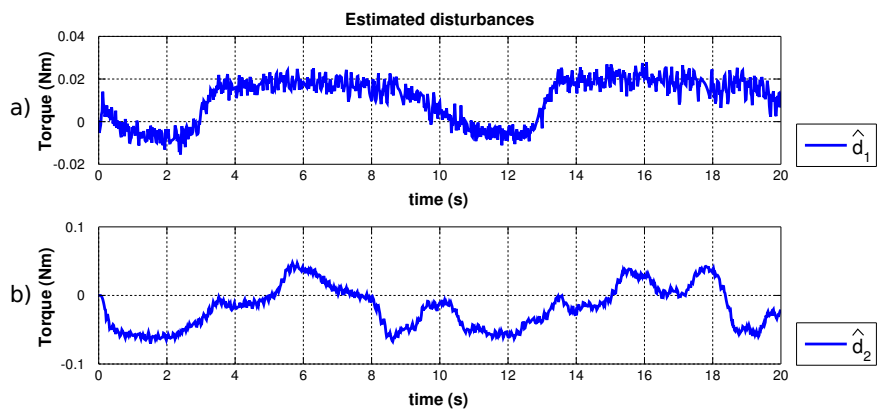

Fig. 7. Experimental results: estimation of the dry frictions $\hat{d}_{1}$ and $\hat{d}_{2}$

\section{E. Discussion}

The performances for the event-triggered strategy with $\sigma=0.9$ are almost equal to the time-triggered case with about $58 \%$ less control updates. $\theta_{3}$ and $\theta_{4}$ look similar in the time-triggered case (Fig. 3) and the event-triggered case (Fig. 5). Nevertheless, the control input changes more abruptly in the event-triggered case. The smaller is $\sigma$, the lower is the frequency of updates but with a worse IAE and IAU performances indexes.

Furthermore, the importance of the disturbance observer is highlighted in Table I, IAE index is three times higher when the dry friction cancellation is not used. The influence of the sampling period on the number of events has been tested in simulation, it appears that the number of events increases as the sampling period decreases.

\section{CONCLUSIONS AND FUTURE WORKS}

This paper concerns the extension and the application of previous works [10] and [4]. A dynamical model of a gyroscope has been detailed with the inclusion of dry friction disturbances. An output-feedback was designed to control the attitude of a gyroscope. A discrete time linear disturbance observer was constructed to ensure zero steady state error while giving an approximation of dry frictions. Furthermore, the control law is event driven. The computation of the event function keeping the system stable has been discussed, this function permits to update the control input in a non periodical way. The whole strategy has been tested on a real-time system for the stabilization of the angular position of a gyroscope, in response to a slow varying reference. The experimental results for event-triggered control was compared to time-triggered one. This comparison emphasises the reduction of control updates while keeping the same performances level. Eventdriven control's result encourages further development of this field.

Future work is based on event-triggered observer to reduce data communication in both ways. One interesting point would also be to point out the influence of the sampling period on the number of events and on the overall performances. Another idea would be to consider delays in the system's model.

\section{ACKNOWLEDGMENT}

This work has been partially supported by the LabEx PERSYVAL-Lab (ANR-11-LABX-0025).

\section{REFERENCES}

[1] Educational control products, control moment gyroscope (model 750). http://www.ecpsystems.com/.

[2] K.-E. Årzén. A simple event-based PID controller. In Preprints 14th World Congress of IFAC, volume Q, pages 423-428, Beijing, P.R. China, Jan 1999.

[3] K. Astrom and B. Bernhardsson. Comparison of Riemann and Lebesgue sampling for first order stochastic systems. In Proceedings of the 41st IEEE Conference on Decision and Control, 2002, volume 2, pages 2011-2016 vol.2, 2002.

[4] S. Durand, B. Boisseau, J. Martinez-Molina, N. Marchand, and T. Raharijaona. Event-based LQR with integral action. In Emerging Technology and Factory Automation (ETFA), IEEE, Sept 2014.

[5] S. Durand and N. Marchand. Further results on event-based PID controller. In European Control Conference (ECC'09), Budapest, Hongrie, Aug. 2009.

[6] S. Durand, N. Marchand, and F. Guerrero Castellanos. Simple Lyapunov sampling for event-driven control. In Preprints of the 18th IFAC World Congress, pages 8724-8730, Milan, Italie, Aug 2011.

[7] W. Heemels, M. Donkers, and A. Teel. Periodic event-triggered control for linear systems. Transactions on Automatic Control, IEEE, 58(4):847-861, April 2013.

[8] W. Heemels, K. Johansson, and P. Tabuada. An introduction to event-triggered and self-triggered control. In 2012 IEEE 51st Annual Conference on Decision and Control (CDC), pages 3270-3285, 2012.

[9] H. Kopetz. Internet of Things. In Real-Time Systems, Real-Time Systems Series, pages 307-323. Springer US, 2011.

[10] N. Marchand, S. Durand, and J. F. Guerrero Castellanos. A general formula for event-based stabilization of nonlinear systems. IEEE Transactions on Automatic Control, 58(5):1332-1337, May 2013.

[11] N. Marchand, J. J. Martinez Molina, S. Durand, and J. F. GuerreroCastellanos. Lyapunov event-triggered control: A new event strategy based on the control. In 9th IFAC Symposium on Nonlinear Control Systems (NOLCOS 2013), pages 324-328, Toulouse, France, Sep 2013.

[12] J. Martinez Molina and H. Khennouf. Approche d'état pour la commande optimale d'un système gyroscopique. In 9e Colloque sur l'Enseignement des Technologies et des Sciences de l'Information et des Systèmes (CETSIS 2011), 2011.

[13] J. Sánchez, M. A. Guarnes, and S. Dormido. On the application of different event-based sampling strategies to the control of a simple industrial process. Sensors, 9(9):6795-6818, aug 2009.

[14] A. Seuret, C. Prieur, and N. Marchand. Stability of non-linear systems by means of event-triggered sampling algorithms. IMA Journal of Mathematical Control and Information, 31(3):415-433, 2014.

[15] P. Tabuada. Event-triggered real-time scheduling of stabilizing control tasks. IEEE Transactions on Automatic Control, 52(9):1680-1685, 2007.

[16] M. Velasco, P. Marti, and E. Bini. On Lyapunov sampling for eventdriven controllers. In Proceedings of the 48th IEEE Conference on Decision and Control, 2009 held jointly with the 2009 28th Chinese Control Conference. CDC/CCC 2009, pages 6238-6243, Shangai, P.R. China, Dec 2009. 